\title{
Correcting for bias in estimation of quantitative trait loci effects
}

\author{
Joel Ira WeLleR*, Meital SHLEZINGER, Micha RoN \\ Institute of Animal Sciences, ARO, The Volcani Center, Bet Dagan 50250, Israel
}

(Received 28 February 2005; accepted 4 May 2005)

\begin{abstract}
Estimates of quantitative trait loci (QTL) effects derived from complete genome scans are biased, if no assumptions are made about the distribution of QTL effects. Bias should be reduced if estimates are derived by maximum likelihood, with the QTL effects sampled from a known distribution. The parameters of the distributions of QTL effects for nine economic traits in dairy cattle were estimated from a daughter design analysis of the Israeli Holstein population including 490 marker-by-sire contrasts. A separate gamma distribution was derived for each trait. Estimates for both the $\alpha$ and $\beta$ parameters and their SE decreased as a function of heritability. The maximum likelihood estimates derived for the individual QTL effects using the gamma distributions for each trait were regressed relative to the least squares estimates, but the regression factor decreased as a function of the least squares estimate. On simulated data, the mean of least squares estimates for effects with nominal $1 \%$ significance was more than twice the simulated values, while the mean of the maximum likelihood estimates was slightly lower than the mean of the simulated values. The coefficient of determination for the maximum likelihood estimates was five-fold the corresponding value for the least squares estimates.
\end{abstract}

genetic markers / quantitative trait loci / genome scans / maximum likelihood / dairy cattle

\section{INTRODUCTION}

Many studies have shown that individual quantitative trait loci (QTL) can be detected and mapped in commercial animal populations with the aid of genetic markers. The daughter and granddaughter designs are most appropriate for analysis of dairy cattle populations [24]. With the advent of DNA microsatellites it became possible to scan the entire genome for QTL for all traits of economic interest. At least three complete genome QTL scans by the granddaughter design have been completed for the US Holstein population. In addition, granddaughter design genome scans have been completed for Canadian, Dutch, French, and German Holsteins, Finish Ayshire, Norwegian cattle, and

*Corresponding author: weller@agri.huji.ac.il 
French Normande and Montbeliarde cattle. These studies are summarized at: http://www.vetsci.usyd.edu.au/reprogen/QTL_Map/.

Beavis [2] first noted that the estimates for statistically significant effects will be biased due to selection. Georges et al. [7] and Beavis [3] showed that bias will be greater for small effects. Although large QTL effects will be deemed significant in any case, small or marginal effects will be denoted "significant" only if the estimate is larger than the actual effect, because of a large positive error term.

Allison et al. [1] and Broman [4] noted that the estimates of the nonsignificant effects will also be biased. Generally, either maximum likelihood (ML) or least squares (LS) methodology is used to estimate QTL effects, which in either event are considered fixed. That is, no assumptions are made with respect to the statistical distribution of QTL effects. Since in nearly all QTL analyses only the absolute value of the effect is considered, LS or ML estimates that do not account for the distribution of QTL effects will be inflated due to nonzero residuals. Assuming that the residual and the actual QTL effects are uncorrelated, the variance of the LS estimates will be equal to the sum of the residual and true QTL effect variance. Thus positive values for QTL effects will be obtained even in the absence of a segregating QTL. Goring et al. [8] noted an additional source of bias in genome scans. When a LOD score or regression effect is maximized over many pointwise tests, the locus-specific effect-size estimate is also maximized. Several studies have proposed methods that deal with some of these problems $[1,20,26]$.

The "multiple comparison problem" is an additional source of bias, not considered in these studies. If several families are analyzed for a number of traits based on a battery of markers covering nearly the entire genome, thousands of statistical tests are performed, and standard levels for null hypothesis rejection are meaningless. Several different solutions have been proposed, summarized by Fernando et al. [6] and Weller et al. [25]. In addition to the question of appropriate rejection thresholds for null hypotheses, the large number of comparisons will also inflate the estimated effects for those contrasts deemed "significant", as described previously.

As the sample size increases, the residual variance for the estimated QTL effect decreases. In best linear unbiased prediction (BLUP) additive genetic effects are considered random, and are regressed as functions of the quantity of data and the ratio of the genetic to residual variance. In BLUP both the genetic and residual effects are assumed to have normal distributions [10].

Fernando and Grossman [5] first proposed to consider QTL as random effects sampled from a distribution with known parameters. If many QTL are 
analyzed jointly it should be possible to estimate both the QTL effects and the parameters of the distribution of QTL effects. However this requires assumptions about the nature of distribution of QTL effects, and a joint analysis of a very large data set (at least several hundred estimated QTL effects, each estimated from several hundred individual quantitative trait and genotype observations) for both the parameters of the QTL distribution and the individual QTL effects.

Allison et al. [1] proposed empirical Bayes approaches to deal with the problem of bias in QTL estimates, but did not present the details. Several studies have considered the question of the appropriate distribution for QTL effects, and in nearly all cases a single-sided distribution was assumed [22]. That is, the QTL effect was assumed to vary from zero to infinity. Hoeschele and Van Raden [11] assumed an exponential distribution of QTL effects. This distribution varies from zero to infinity, and is a function of only one parameter. They also estimated the effect for a simulated QTL using both ML and Bayesian methodology. As expected, Bayesian estimates of the QTL effect were regressed relative to the ML estimates, which made no prior assumptions about the distribution of QTL effects. Hoeschele and Van Raden [12] varied the sample size in their simulations, but not the magnitude of the QTL.

Hayes and Goddard [9] estimated the distribution of QTL effects for cattle and swine by combining the results from several studies. They assumed a gamma distribution for the QTL effects. Like the exponential distribution, the gamma distribution varies from zero to infinity, but is more flexible, because it is a function of two parameters. A single gamma distribution was assumed for each species, even though several different traits were analyzed. Hayes and Goddard's [9] dairy cattle analysis was based on the QTL estimates from three granddaughter design analyses, considering only "significant" effects. Thus a truncated gamma distribution was assumed. The dairy cattle data set included only 50 observations, because each QTL estimate was considered a single data point.

The assumption of a single distribution per species for all segregating QTL is problematic, because the traits analyzed have different heritabilities and were exposed to differing selection intensities. For example, dairy cattle have been highly selected for milk production for 50 years, while there has been virtually no direct selection for fat concentration. In addition, it should be possible to more accurately estimate the QTL distribution parameters if both significant and nonsignificant effects are included in the analysis.

We recently completed a daughter design genome scan of the Israeli Holstein population for nine economic traits [18]. Although it is not practical 
to estimate jointly both the distribution of the QTL effects and the individual effects from a complete genome scan, it is possible to first estimate the distribution of the QTL effects, and then estimate each of the individual random QTL effects with their distributions assumed known. The objectives of the current study were to estimate the parameters of the distributions of QTL effects in the Israeli Holstein population, under the assumption of a separate gamma distribution for each of the nine traits analyzed, and to use these distributions to derive ML estimates of a sample of QTL effects with the QTL effects considered random with known distributions. (From this point onward, "ML estimates" will refer only to estimates derived under the assumption that the QTL effects are randomly sampled from a known distribution.) Finally a simulated genome scan was generated based on the gamma distribution derived for protein percentage, the trait with the highest heritability. QTL estimates derived by ML and LS were compared to each other and to the simulated values.

\section{MATERIALS AND METHODS}

\subsection{Data}

Eleven Israeli Holstein families including 5221 cows were analyzed by a daughter design for nine economic traits; milk, fat and protein production, fat and protein percentage, somatic cell score (SCS), herd-life, female fertility, and the Israeli breeding index (PD01), computed as follows for each animal:

PD01 $=-0.22 \times($ milk $)+8.5 \times($ fat $)+31 \times($ protein $)-300 \times($ SCS $)+26 \times($ fertility $)$

where milk, fat, protein, SCS, and fertility are the genetic evaluations for the animal. Details of the analysis were presented by Ron et al. [18]. The basic genome scan included 73 microsatellites, but a QTL effect could be estimated only for sires heterozygous for a specific marker. Preliminary analysis was by ANOVA of the cows' genetic evaluation for each trait, with the marker effect nested within sire. All cows included in the analysis had genetic evaluations for the five milk production traits, but a few cows were lacking values for the secondary traits; SCS, herd-life, fertility, and PD01. Genotypes were considered "informative" if the sire was heterozygous, and the genotype of the daughter was different from the genotype of the sire [16]. Families with significant effects were genotyped for an additional 35 markers linked to the markers with significant effects, for a total of 108 markers. There were 98287 informative genotypes, and 490 marker-by-sire contrasts; for a mean of 4.5 contrasts per 
marker, and 201 informative genotypes per contrast. The number of informative genotypes per contrast varied from 83 to 591. A QTL effect for each of the nine traits was estimated for each contrast, for a total of 4410 QTL effects. All effects were normalized to equal variance by analysis of the $t$-values.

The greatest QTL effect was detected for the effect of protein percent near the middle of chromosome 6 [17]. Two sire families, 2278 and 3099, were heterozygous for this QTL. Daughters of sire 2278 were selected for further analysis, because this family was larger. The daughters were genotyped for 11 markers on this chromosome, and the highest test statistic was obtained at marker BM143. A total of 393 daughters were informative for this marker, and slightly less had evaluations for the secondary traits (Tab. III). A total of 683 daughters with evaluations for the production traits were informative for at least one marker on this chromosome. Of these, 641 cows had evaluations for all of nine traits, and this subset was used for the analysis of the secondary traits. The probability to obtain either paternal allele at the assumed QTL location was computed for each daughter as described by Knott et al. [14] for these two samples.

For ML analysis of the individual records, it was necessary to normalize the individual records to the same scale used to estimate the gamma distributions; the scale of $t$-values. The genetic evaluations were normalized first by division by the standard deviation of the genetic evaluations for each trait. For the analysis of the daughters genotyped for BM143, the values were then subtracted from the mean of the means of the two genotype classes. The contrasts were then normalized to a $t$-value by division by the standard error (SE) of the contrast computed as: $\left(1 / n_{1}+1 / n_{2}\right)^{1 / 2}$, where $n_{1}$ and $n_{2}$ are the number of daughters that received the paternal alleles with positive and negative effects, respectively. For the production traits, there were 221 and 172 cows with the two paternal alleles, respectively. Thus the SE of the contrast was 0.102 .

For analysis of the entire chromosome, the genetic evaluations were normalized by subtracting the grand mean and dividing by the SE of the regression of the trait value on the probability that the daughter received the "positive" QTL allele from her sire. Among the traits analyzed, the regression SE increased as the QTL effect decreased. The highest SE was used for normalization. The production and non-production traits were normalized separately, because of the difference in the sample sizes. The SE values used for normalization were 0.082 for the production traits, and 0.085 for the secondary traits. 


\subsection{Estimation of the parameters for the QTL distributions}

Following Hayes and Goddard [9], the QTL were assumed to follow a gamma distribution with scaling parameter $\alpha$ and shape parameter $\beta$. Defining $x$ as the absolute difference between the substitution effects of the two paternal QTL alleles, $g(x)$ the distribution of $x$ is:

$$
g(x)=\frac{\alpha^{\beta} x^{\beta-1} e^{-\alpha x}}{\int_{0}^{\infty} t^{\beta-1} e^{-t} \mathrm{~d} t} .
$$

The mode of the gamma distribution is $(\beta-1) / \alpha$. If $\beta<1$, the mode of the distribution will be at zero. A normal distribution is assumed for the residuals. Thus the ordinate of observed QTL effect, $\hat{x}_{i}$, given the actual effect, $\mathrm{n}\left(\hat{x}_{i} \mid x\right)$, will be:

$$
n\left(\hat{x}_{i} \mid x\right)=\frac{1}{\sqrt{2 \pi \sigma_{x}^{2}}} e^{-\left(\frac{\left(\hat{x}_{i}-x\right)^{2}}{2 \sigma_{x}^{2}}\right)}
$$

where $\sigma_{x}=$ the SE of the estimated QTL effect. This value will vary as a function of the experiment size.

As noted by Hayes and Goddard [9], although the QTL effect is assumed always to be positive, the residual can be either positive or negative. Thus the density for $\hat{x}_{i}, f\left(\hat{x}_{i}\right)$ is computed as follows:

$$
f\left(\hat{x}_{i}\right)=\int_{0}^{\infty} n\left(\hat{x}_{i} \mid x\right) g(x) \mathrm{d} x+\int_{0}^{\infty} n\left(-\hat{x}_{i} \mid x\right) g(x) \mathrm{d} x .
$$

Unlike Hayes and Goddard [9] we did not truncate the likelihood, because all contrasts were included. The log likelihood for the distribution of the QTL effects, $\log L(x)$, summed over all observed effects for each trait is:

$$
\log L(x)=\sum_{i=1}^{I} \log \left[f\left(\hat{x}_{i}\right)\right]
$$

where $I$ is the total number of estimated QTL effects per trait. Numerical integration was used to compute the density function, and $\log L(x)$ was maximized relative to $\alpha, \beta$, and $\sigma_{x}$ for each trait by a grid search for the three parameters. Since the analysis was performed on the $t$-values of the contrasts, $\sigma_{x}$ should be approximately equal to unity. Therefore, $\log L$ was also maximized for $\alpha$ and $\beta$, with $\sigma_{x}$ fixed at unity. 
In both cases, the prediction error variances of the parameter estimates were estimated by the negative of the inverse of the matrix of second derivatives of $\log L$ at its maximum. The matrix of second derivatives was estimated numerically.

In addition the following model was tested which, similar to the "BayesB" method of Meuwissen et al. [15], considers the possibility that only a fraction of the marker contrasts were associated with segregating QTL:

$$
f\left(\hat{x}_{i}\right)=P\left(\int_{0}^{\infty} n\left(\hat{x}_{i} \mid x\right) g(x) \mathrm{d} x+\int_{0}^{\infty} n\left(-\hat{x}_{i} \mid x\right) g(x) \mathrm{d} x\right)+2(1-P)\left(\int_{0}^{\infty} n\left(\hat{x}_{i} \mid x\right) \mathrm{d} x\right)
$$

where $P=$ the fraction of marker contrasts, and the other terms are as defined previously. This model was applied to two traits, protein percentage and fertility, with $\sigma_{x}$ fixed at unity.

\subsection{Estimation of QTL effects}

The likelihood of the individual QTL effects given the distribution of QTL effects for a given trait, $L(y)$ will first be computed under the assumption that QTL genotype has been determined for each individual. This was assumed to be the case for the analysis of the effect associated with marker BM143. For the daughter design, only the paternal allele is considered, and the progeny will be divided into two groups; the $J_{1}$ individuals that received the positive paternal QTL allele, and the $J_{2}$ individuals that received the negative paternal allele. $L(y)$ is then computed as follows:

$$
L(y)=g\left(x_{i} \mid \alpha, \beta\right) \prod_{j=1}^{J_{1}}\left(\frac{1}{\sqrt{2 \pi \sigma_{y}^{2}}} e^{-\left(\frac{\left(y_{j}-0.5 x_{i}\right)^{2}}{2 \sigma_{y}^{2}}\right)}\right) \prod_{j=J_{1}+1}^{J}\left(\frac{1}{\sqrt{2 \pi \sigma_{y}^{2}}} e^{-\left(\frac{\left(y_{j}+0.5 x_{i}\right)^{2}}{2 \sigma_{y}^{2}}\right)}\right)
$$

where $x_{i}=$ the effect for QTL $i, y_{j}=$ standardized record of individual $j$, $J=J_{1}+J_{2}=$ the total number of individuals genotyped for the QTL, $\sigma_{y}^{2}$ is the residual variance of the individual records, and the other terms are as described previously. Since the observations were normalized by subtraction of the mean of the two means, it is not necessary to include a mean effect in the likelihood. Since $\alpha$ and $\beta$ are assumed known, this likelihood was maximized only relative to $x_{i}$ and $\sigma_{y}$. 
$\log L(y)$, the log likelihood, with terms including only constants deleted is computed as follows:

$$
\begin{aligned}
\log L(y)=(\beta-1) \log \left(x_{i}\right)-a x_{i} & -J\left(\log \left(\sigma_{y}\right)\right)-\left[1 /\left(2 \sigma_{y}^{2}\right)\right] \\
\times & {\left[\sum_{j=1}^{J_{1}}\left(y_{j}-0.5 x_{i}\right)^{2}+\sum_{j=J_{1}+1}^{J}\left(y_{j}+0.5 x_{i}\right)^{2}\right] . }
\end{aligned}
$$

Solutions were obtained by a one-dimensional search with respect to $x_{i}$. At each value of $x_{i}$, the $\mathrm{ML}$ value for $\sigma_{y}$ was determined by solving for $\partial[\log L(y)] / \partial \sigma_{y}=0$ as follows:

$$
\sigma_{y}^{2}=\frac{\sum_{j=1}^{J_{1}}\left(y_{j}-0.5 x_{i}\right)^{2}+\sum_{j=J_{1}+1}^{J}\left(y_{j}+0.5 x_{i}\right)^{2}}{J} .
$$

In the interval mapping QTL analysis, the genotype of each individual with respect to the QTL is not known with certainty. In this case $L(y)$ is computed as follows:

$$
L(y)=g\left(x_{i} \mid \alpha, \beta\right) \prod_{j=1}^{J}\left[\left(\frac{p_{j}}{\sqrt{2 \pi \sigma_{y}^{2}}} e^{-\left(\frac{\left(y_{j}-0.5 x_{i}\right)^{2}}{2 \sigma_{y}^{2}}\right)}\right)+\left(\frac{1-p_{j}}{\sqrt{2 \pi \sigma_{y}^{2}}} e^{-\left(\frac{\left(y_{j}+0.5 x_{i}\right)^{2}}{2 \sigma_{y}^{2}}\right)}\right)\right]
$$

where $p_{j}$ is the probability that the progeny received the positive paternal allele, given its marker genotype. This likelihood was solved for $x_{i}$ and $\sigma_{y}$ by a two-dimensional grid search.

The prediction error variances of the QTL estimates were estimated by two methods. First, from the inverse of the two-by-two matrix of second derivatives for $x_{i}$ and $\sigma_{y}$, as described for the parameters of the gamma distribution. These were denoted "empirical" values because the second derivatives were derived numerically. The second method applied the assumption that the minor diagonal elements of the matrix of second derivatives of $\log L(y)$ are small relative to the major diagonal elements. Under this assumption, $-1 /\left[\partial^{2}[\log L(y)] / \partial x_{i}^{2}\right]$ will be approximately equal to the prediction error variance of $x_{i}$. If the QTL genotype was assumed known without error, $\partial^{2}[\log L(y)] / \partial x_{i}^{2}$ is computed as follows:

$$
\frac{\partial^{2}[\log L(y)]}{\partial x_{i}^{2}}=\frac{1-\beta}{x_{i}^{2}}-\frac{J}{4 \sigma_{y}^{2}} .
$$

For large values of $x_{i}$, the first term on the right-hand side of Eq. (11) tends to zero. Similarly, $\partial^{2}[\log L(y)] / \partial \sigma_{y}^{2}$ can be derived by differentiating Eq. (8) 
twice and substituting from Eq. (9) as follows:

$$
\frac{\partial^{2}[\log L(y)]}{\partial \sigma_{y}^{2}}=-\frac{2 J}{\sigma_{y}^{2}}
$$

which is the value of $\partial^{2}[\log L(y)] /\left[\partial \sigma_{y}^{2}\right]$ for a sample from a normal distribution. For both methods, SE estimates were derived as the square roots of the corresponding prediction error variances.

\subsection{Simulation analysis}

We simulated a daughter design genome scan with 1000 contrasts under the assumption that the true effects were sampled from a gamma distribution with $\alpha$ and $\beta$ values equal to estimates for protein percentage. For each contrast, an effect was simulated by random sampling from this gamma distribution, and a sample of 400 individual records was generated. Each individual had a $50 \%$ chance to receive the positive or the negative QTL allele. A random residual was generated by sampling from a normal distribution with mean zero and a standard deviation of 10. Thus the expected SE for the QTL effect for a balanced sample of 400 individuals will be equal to unity. The trait value for each individual was then computed as the residual $+1 / 2$ the QTL effect for individuals that received the positive allele, and $-1 / 2$ the QTL effect for individuals that received the negative allele. The LS QTL effect was then estimated for each simulated QTL based only on the genotypes and trait records. If the absolute value of the $t$-value was $>2.5$ (a probability of 0.012 for comparison-wise significance) then the QTL effect was also estimated by ML, with the QTL genotypes assumed known. The LS and ML QTL estimates of the significant effects were compared to each other and to the simulated QTL values. Mean squared deviations of both estimates from the simulated values, coefficients of determinations $\left(\mathrm{R}^{2}\right)$, and regressions of the simulated values on the estimates were computed. Standard errors for the ML estimates were computed by the two methods described.

\section{RESULTS}

The ML estimates of the QTL parameters, including $\sigma_{x}$, are given in Table I with their SE. Since, as noted, the mode of the gamma distribution $=(\beta-1) / \alpha$, distributions with $\beta<1$ have modes at $x=0$. This was the case only for fat and protein percent. The first parity trait heritabilities are also given. Hayes 
Table I. Maximum likelihood estimates of the QTL parameters, including $\sigma_{x}$.

\begin{tabular}{lcccc}
\hline & & \multicolumn{3}{c}{ Parameter estimates } \\
\cline { 3 - 5 } & Heritability $^{\mathrm{a}}$ & $\alpha \pm \mathrm{SE}$ & $\beta \pm \mathrm{SE}$ & $\sigma_{x} \pm \mathrm{SE}$ \\
\hline Milk yield & 0.43 & $2.31 \pm 0.60$ & $1.16 \pm 0.31$ & $1.02 \pm 0.10$ \\
Fat yield & 0.38 & $2.45 \pm 0.64$ & $1.18 \pm 0.33$ & $1.01 \pm 0.09$ \\
Protein yield & 0.36 & $3.36 \pm 2.00$ & $1.44 \pm 0.73$ & $0.98 \pm 0.15$ \\
Fat percentage & 0.58 & $2.01 \pm 0.36$ & $0.96 \pm 0.18$ & $1.29 \pm 0.06$ \\
Protein percentage & 0.78 & $1.99 \pm 0.32$ & $0.90 \pm 0.15$ & $1.30 \pm 0.05$ \\
SCS & 0.20 & $4.22 \pm 4.80$ & $1.65 \pm 1.24$ & $1.00 \pm 0.23$ \\
Herd-life $^{\mathrm{b}}$ & 0.11 & $7.50 \pm 9.78$ & $5.86 \pm 5.60$ & $0.81 \pm 0.17$ \\
Fertility $_{\text {PD01 }^{\mathrm{c}}}$ & 0.03 & $8.75 \pm 11.47$ & $5.86 \pm 7.36$ & $0.81 \pm 0.12$ \\
\hline
\end{tabular}

${ }^{a}$ First parity estimates are from Weller and Ezra [23] for all traits, except herd-life and PD01.

${ }^{\mathrm{b}}$ Heritability from Settar and Weller [19]. There was only one record per cow for this trait.

${ }^{c}$ Heritability computed as $\mathbf{b G} \mathbf{b}^{\prime} / \mathbf{b P} \mathbf{b}^{\prime}$ where $\mathbf{b}=$ vector of index coefficients, $\mathrm{G}=$ first parity genetic variance matrix, and $\mathrm{P}=$ first parity phenotypic variance matrix.

and Goddard's [9] estimate of 5.4 for $\alpha$ for dairy cattle is within the range of estimates in Table I, but their estimate of 0.42 for $\beta$ is considerably lower than all the estimates obtained in this study. However they only used "significant" QTL effects to estimate these parameters. The estimates for $\sigma_{x}$ ranged from 0.8 to 1.3 , and increased as a function of the heritabilities. The estimates of $\sigma_{x}$ were significantly different from unity only for fat and protein percentage. Estimates for both $\alpha$ and $\beta$ and their SE were negatively correlated with the heritability. For traits with heritability $<0.25$ confidence intervals for $\alpha$ and $\beta$, estimated as $\pm 2 \mathrm{SE}$, included zero. Thus the estimates derived for these traits are virtually useless, even though the sample sizes were quite large. Surprisingly, this was also the case for protein yield, even though its heritability was 0.36 .

The gamma distributions of QTL effects for protein yield, protein percentage, and fertility are plotted in Figure 1. The distribution of fat percentage was very similar to the distribution of protein percentage, the distribution of herdlife was very similar to the distribution of fertility, and the distributions of the remaining traits were very similar. Therefore distributions are shown only for protein yield, protein percentage, and fertility. Protein percentage, with a mode at zero, had the highest density at high $x$-values; while fertility, with the highest mode, had the lowest density at high $x$-values.

Estimates for $\alpha$ and $\beta$ with $\sigma_{x}$ fixed at unity and their SE are given in Table II. For fat percentage and protein, $\alpha$ values were slightly lower, and $\beta$ values were slightly higher. For the production traits, $\alpha$ and $\beta$ values were nearly the 


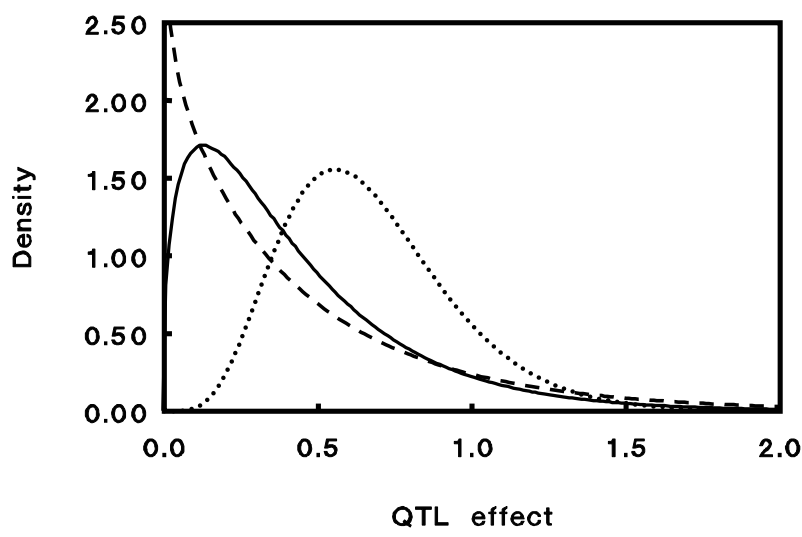

Figure 1. The gamma distributions of the QTL effects. - , protein; --, protein percentage; $\cdots$, female fertility. Trait units are the standard errors of the QTL effects.

Table II. Maximum likelihood estimates of the QTL parameters, with $\sigma_{x}$ fixed at unity.

\begin{tabular}{lcc}
\hline \multirow{2}{*}{ Trait } & \multicolumn{2}{c}{ Parameter estimates } \\
\cline { 2 - 3 } & $\alpha \pm \mathrm{SE}$ & $\beta \pm \mathrm{SE}$ \\
\hline Milk yield & $2.25 \pm 0.49$ & $1.17 \pm 0.32$ \\
Fat yield & $2.40 \pm 0.54$ & $1.18 \pm 0.33$ \\
Protein yield & $3.53 \pm 1.48$ & $1.42 \pm 0.70$ \\
Fat percentage & $1.70 \pm 0.28$ & $1.08 \pm 0.23$ \\
Protein percentage & $1.65 \pm 0.24$ & $0.95 \pm 0.17$ \\
SCS & $4.26 \pm 2.99$ & $1.67 \pm 1.40$ \\
PD01 & $2.49 \pm 0.68$ & $1.32 \pm 0.45$ \\
\hline
\end{tabular}

same as in Table I. This is expected, since the estimates of $\sigma_{x}$ for these traits were all very close to unity. It was not possible to derive parameter estimates for fertility and herd-life with $\sigma_{x}$ fixed at unity. Likelihood values kept increasing even with $\alpha$ and $\beta>20$. This corresponds to the results of Ron et al. [18] that for these traits the number of "significant" effects was virtually equal to the number expected by chance at all significance levels. SE were generally similar to the values in Table I. Based on these SE, none of the parameter estimates in Table II were significantly different from the estimates in Table I.

Comparisons of the observed and expected frequencies of the LS QTL effects are given in Figures 2, 3, and 4 for protein percentage, protein yield, and fertility, respectively. The expected frequencies with $\sigma_{x}$ fixed at unity and with $\sigma_{x}$ estimated are given in Figure 2 for protein percentage. There were 10 observations with $x>4$ (2\% of the total) not shown in the figure. The expected density at $x=0$ was slightly lower with the estimated value of $\sigma_{x}$, while the density at intermediate densities was higher. For protein yield the two 

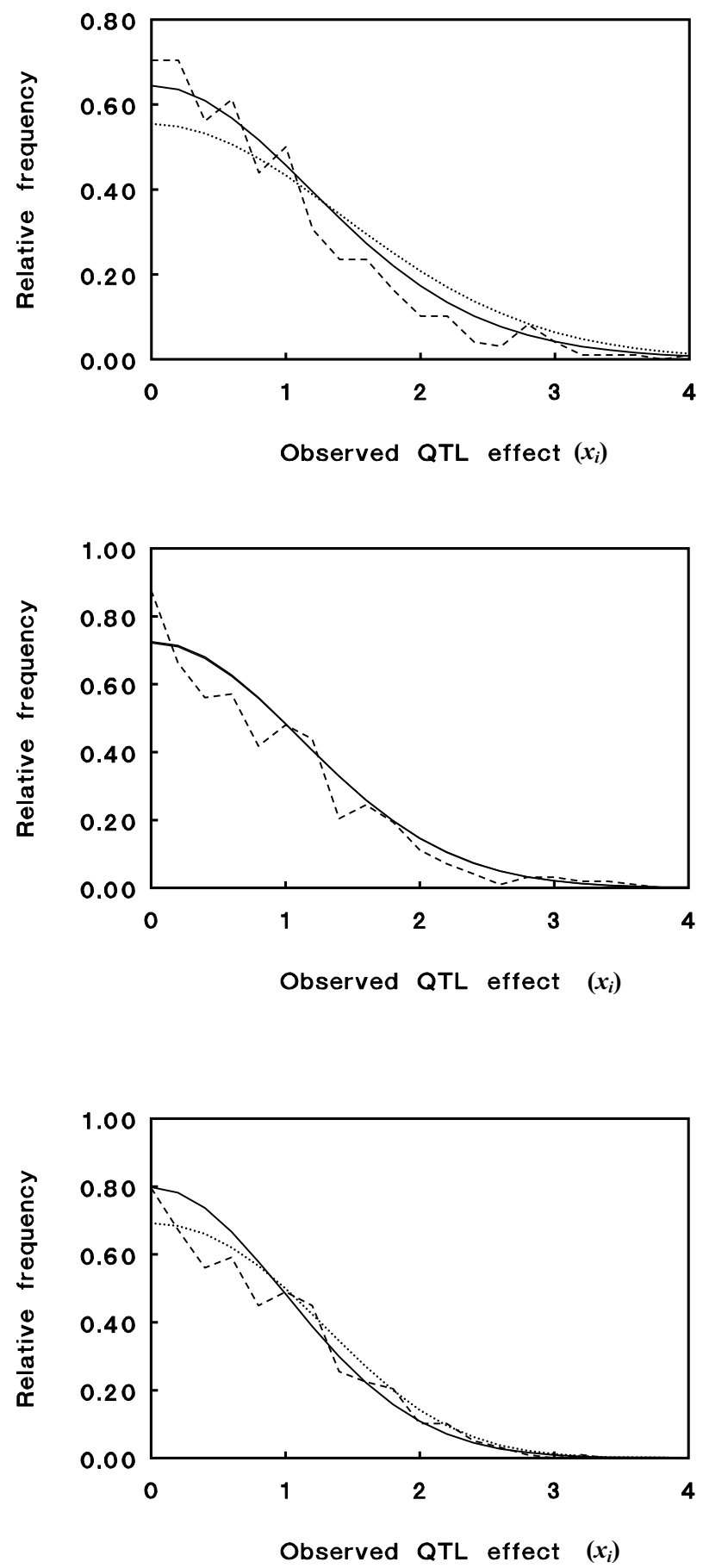

Figure 2. Comparison of the expected and observed frequency distribution of the least squares QTL effects for protein percentage. -, expected frequency with $\sigma_{x}$ fixed at unity; $\cdots$, expected frequency with $\sigma_{x}$ estimated; --, observed frequency. Trait units are the standard errors of the QTL effects.

Figure 3. Comparison of the expected and observed frequency distribution of least squares QTL effects for protein yield. - , expected frequency with $\sigma_{x}$ fixed at unity; - -, observed frequency. Trait units are the standard errors of the QTL effects.

Figure 4. Comparison of the expected and observed frequency distribution of least squares QTL effects for female fertility. - , expected frequency with no segregating QTL; $\cdots$, expected frequency with segregating QTL and $\sigma_{x}$ estimated; - -, observed frequency. Trait units are the standard errors of the QTL effects. 
Table III. Maximum likelihood and least squares estimates of the QTL effects on chromosome 6 for sire 2278 considering only daughters with informative genotypes for BM143.

\begin{tabular}{lcccccc}
\hline & & \multicolumn{5}{c}{ Estimates } \\
\hline & Number & \multirow{2}{*}{-test } & \multicolumn{4}{c}{ QTL effect } \\
\cline { 4 - 6 } Trait & 393 & $10^{-5}$ & 4.55 & $0.59 \pm 0.73$ & $(0.82)$ & $9.76 \pm 0.35$ \\
\hline Milk yield & 393 & 0.0008 & 3.55 & $0.24 \pm 0.37$ & $(0.49)$ & $9.80 \pm 0.35$ \\
Fat yield & 393 & 0.0026 & 2.97 & $0.22 \pm 0.23$ & $(0.31)$ & $9.81 \pm 0.35$ \\
Protein yield & 393 & $10^{-10}$ & 7.34 & $3.78 \pm 0.97$ & $(0.94)$ & $9.29 \pm 0.34$ \\
Fat percentage & 393 & $10^{-14}$ & 10.07 & $7.05 \pm 0.89$ & $(0.87)$ & $8.59 \pm 0.31$ \\
Protein percentage & 392 & NS & 1.58 & $0.18 \pm 0.16$ & $(0.22)$ & $9.82 \pm 0.35$ \\
SCS & 378 & NS & 0.22 & $0.21 \pm 0.08$ & $(0.09)$ & $9.82 \pm 0.36$ \\
Herd-life & 384 & NS & 1.93 & $0.60 \pm 0.19$ & $(0.26)$ & $9.79 \pm 0.35$ \\
Fertility & 383 & $10^{-7}$ & 4.68 & $0.48 \pm 0.55$ & $(0.68)$ & $9.77 \pm 0.36$ \\
PD01 & & & & & & \\
\hline
\end{tabular}

a The empirical standard errors are listed next to the QTL effect estimate, and the standard errors derived from Eq. (10) are given in parentheses.

expected functions were nearly identical, and thus only the function with $\sigma_{x}$ fixed is shown. For fertility, the expected frequencies were computed with the estimated value for $\sigma_{x}$ from Table I, and based on the assumption of no segregating QTL. At $x=0$ the expected density with a gamma distribution of QTL was slightly lower than the distribution that assumed no segregating QTL, while the density at intermediate $x$-values was higher. For all three traits there was good correspondence between the expected and observed QTL LS estimate distributions.

For the analysis by Eq. (7) maximum likelihood was obtained for both protein percentage and fertility with $P=1$, that is under the assumption that some segregating QTL effect is associated with all marker contrasts.

ML estimates of the QTL parameters, assuming complete linkage between the QTL and the genetic marker BM143 on chromosome 6 are given in Table III. These estimates were derived using the gamma distributions presented in Table I. The sample sizes are also given, but they were all very similar. For the selection index, the regression factor is a direct function of the LS estimate for all samples of the same size. This was not the case for the ML estimates. The ML estimates of the significant effects were all highly regressed relative to the LS estimates, but the regression factor was greater for the smaller effects. For fat and protein yield the regression factor was $>10$ fold, but only two-fold for fat percentage, and 1.5 for protein percentage. Note that 
Table IV. Maximum likelihood and least squares estimates of the QTL effects on chromosome 6 including all daughters of sire 2278 with at least one informative marker.

\begin{tabular}{lcccc}
\hline & & \multicolumn{3}{c}{ Estimates } \\
\hline Trait & Number & \multicolumn{3}{c}{ QTL effect } \\
\cline { 3 - 4 } & of cows & LS & ML \pm SE & $\sigma_{y} \pm \mathrm{SE}$ \\
\hline Milk yield & 683 & 4.16 & $2.00 \pm 1.01$ & $11.80 \pm 0.35$ \\
Fat yield & 683 & 3.75 & $1.50 \pm 0.97$ & $11.71 \pm 0.35$ \\
Protein yield & 683 & 3.58 & $0.81 \pm 0.77$ & $11.74 \pm 0.35$ \\
Fat percentage & 683 & 7.62 & $5.62 \pm 0.96$ & $11.26 \pm 0.34$ \\
Protein percentage & 683 & 10.64 & $8.86 \pm 0.90$ & $10.65 \pm 0.32$ \\
SCS & 641 & 1.55 & $0.23 \pm 0.23$ & $11.78 \pm 0.33$ \\
Herd-life & 641 & 0.19 & $0.48 \pm 0.25$ & $11.79 \pm 0.33$ \\
Fertility & 641 & 1.15 & $0.59 \pm 0.26$ & $11.78 \pm 0.33$ \\
PD01 & 641 & 5.36 & $2.88 \pm 0.97$ & $11.75 \pm 0.33$ \\
\hline
\end{tabular}

for herd-life there was virtually no regression of the ML estimate, because the LS estimate was also quite low. Thus, as expected, regression was the greatest for moderately significant effects. All the estimates of $\sigma_{y}$ were very similar, and quite close to the expected value derived in the Methods section, that is $1 / 0.102=9.8$. The $\sigma_{y}$ SE estimates were very similar for all the traits, and close to 0.35 . This was also the value obtained from Eq. (12). The empirical SE for the QTL effect varied from 0.08 for herd-life to 0.97 for fat percentage, and were generally close to the SE estimates derived from Eq. (11) given in parenthesis. As expected from the $\beta$ values, SE were higher for the traits with higher heritabilities.

ML estimates of the QTL effects and their SE for all animals with at least one informative marker on chromosome 6 are given in Table IV. Sample sizes are also given. The ML estimates were derived from Eq. (10) and the LS estimates were derived by interval mapping [14]. The number of records in Table IV was nearly double the number of records in Table III. The LS estimates were very similar to the LS estimates in Table III, while all the ML estimates for significant effects were larger. The ML estimates as a function of the LS estimates are plotted in Figure 5. The exponential regression curve is also shown. As expected, the regression factor decreased as the LS effect increased. For milk yield the regression factor was 2, while for protein percentage the regression factor was $<1.2$. For herd-life, the ML estimate was more than twice the LS estimate. This is because the mode of the gamma distribution 


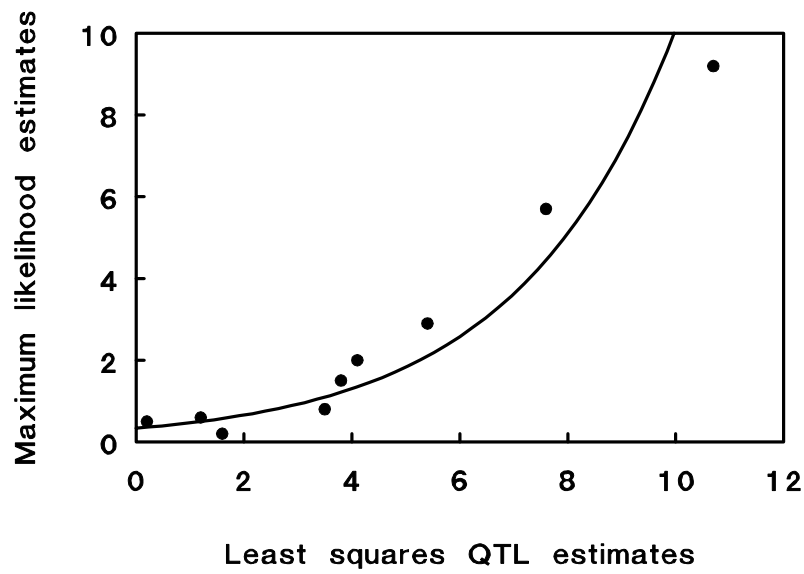

Figure 5. Maximum likelihood estimates as a function of the least squares estimates for the QTL on chromosome six. Trait units are the standard errors of the QTL effects.

for this trait $=(\beta-1) / \alpha=0.648>$ the LS estimate. However, neither estimate was significantly different from zero. The estimates of $\sigma_{y}$ were close to the expected values for the sample sizes as given in the Methods section. Protein percentage had the lowest estimate of $\sigma_{y}$, because a larger fraction of the total variance was absorbed by the QTL effect. As in Table III, the SE of $\sigma_{y}$ in Table IV were very similar for all the traits, and had nearly the same values as in Table III, 0.35; even though the sample size was nearly double. Again as in Table III, the SE for the QTL effect varied widely, from 0.23 for SCS to 1 for milk yield. Surprisingly, the SE were on the average slightly larger in Table IV, even though the sample sizes were larger. However, this analysis also accounted for uncertainty with respect to the QTL genotype, and as shown in Eq. (11), the SE is also a function of the ML estimate.

The results of the simulated genome scan are summarized in Table V. The mean of the 1000 simulated contrasts was 0.566 , as compared to the expected value of $\beta / \alpha=0.95 / 1.65=0.576$ [9]. There were 54 contrasts with $t$-values $>2.5$, as compared to $1000 \times 0.012=12$ expected purely by chance. Thus the false discovery rate $=0.22$ [25]. Similar to the results of Goring et al. [8] for LOD scores, the LS estimates were highly biased, with a mean value of 3.04, as compared to 1.45 for the simulated values. The mean of the ML estimates was 1.26, which is much closer to the simulated values. The standard deviation of the ML estimates was slightly higher than the LS estimates, although both standard deviations were considerably lower than the standard deviation of the simulated effects. The LS and ML estimates for $\sigma_{y}$ were very similar, and both were very close to the simulated value of 10 . The standard deviations of the estimates for $\sigma_{y}$ were both 0.35 , which is also the simulated value, and the value obtained from Eq. (12) for a sample of 400 individuals. 
Table V. Comparison of the simulated QTL values and the least squares and maximum likelihood estimates (54 observations).

\begin{tabular}{lccc}
\hline & $\begin{array}{c}\text { Simulated } \\
\text { value }\end{array}$ & LS estimates & ML estimates \\
\hline $\begin{array}{l}x_{i} \text {, mean } \pm \text { standard } \\
\text { deviation }\end{array}$ & $1.451 \pm 0.896$ & $3.038 \pm 0.449$ & $1.263 \pm 0.543$ \\
$\begin{array}{l}\text { Range for } x_{i} \\
\text { (minimum - maximum) }\end{array}$ & $0.015-2.970$ & $2.519-4.298$ & $0.010-2.663$ \\
$\sigma_{y}$, mean \pm standard deviation & $10.000 \pm 0.353$ & $10.002 \pm 0.353$ & $10.027 \pm 0.357$ \\
$\begin{array}{l}\text { Coefficient of determination } \\
\left(\mathrm{R}^{2}\right) \text { for the simulated value } \\
\text { of } x_{i}\end{array}$ & & 0.014 & 0.075 \\
$\begin{array}{l}\text { Regression of simulated } \\
\text { value for } x_{i} \text { on the estimate } \\
\pm \text { SE }\end{array}$ & & \\
$\begin{array}{l}\text { Mean squared deviation of } \\
\text { estimated } x_{i} \text { from the } \\
\text { simulated value }\end{array}$ & & & \\
\hline
\end{tabular}

This is also very close to the estimated SE for $\sigma_{y}$ in Table III for nearly the same sample size.

The $\mathrm{R}^{2}$ of the simulated values was more than five-fold for the ML estimates, as compared to the LS estimates, but both were $<0.1$. For an estimate to be unbiased, the regression of the true value on the estimate should be unity [21]. Both regressions of the simulated values on the estimates were $<0.5$, but the regression on the ML estimate was nearly double the regression of the LS estimate. SE of both regressions were quite large though.

The mean squared deviation of the ML estimates from the simulated value, 0.85, was one fourth of the corresponding value for the LS estimates, 3.41. Under the null hypothesis of no segregating QTL, the SE of the LS estimates should be equal to unity by definition. With segregating QTL, the deviation between the actual value and the estimate includes both sampling variation of the QTL effect and variation in the actual effect. The mean of the computed $\mathrm{SE}$ was 1.02, which overestimated the square root of the mean squared deviation, but is close to the value of 0.9 obtained in the analysis of actual data for protein percentage. Solving for $\partial^{2}[\log L(y)] / \partial x_{i}^{2}$ using the mean estimates for $x_{i}$ and $\sigma_{y}$ gives -1.03 for a mean SE of 0.97 , which is very close to the value obtained by numerical calculation. The SE estimates were very constant with a standard deviation of 0.04 . This is as expected, because, except 


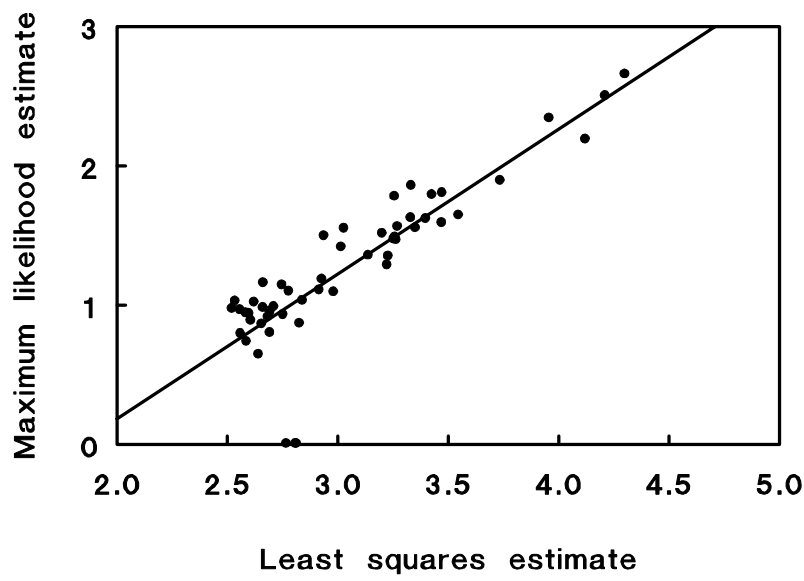

Figure 6. Maximum likelihood estimates of QTL effects as a function of the least squares estimates for the simulated data.

for very low estimates of $x_{i}$, the first term of Eq. (11) will be negligible with respect to the second term, which is nearly constant. The computed SE can be considered "conservative" in that marginally significant differences will be considered nonsignificant.

The ML QTL estimates as a function of the LS estimates are plotted in Figure 6. All the LS estimates are $>2.5$, because this was the selection criteria. Although the correlation between the two estimates was 0.86 , the ML estimates were not a direct function of the LS estimates. The regression value was 1.04. Even though sample sizes were the same for each contrast, the same LS estimates gave differing ML estimates. Three contrasts with LS estimated > 2.5 gave ML estimates of nearly zero (two overlap in the figure). The simulated values as a function of the LS and ML estimates and the regressions are plotted in Figures 7 and 8 . As can be seen from the minimum and maximum values given in Table V, the ML estimates cover nearly the entire range of simulated values. Of the seven contrasts with simulated values $<0.3$, the ML algorithm generated similar estimates for three observations (two overlap in the figure near the origin).

\section{DISCUSSION}

The estimation of the QTL distributions was based on several assumptions that will now be addressed in detail. Similar to Fernando and Grossman [5], who also assumed random QTL effects, we assumed an individual QTL effect for each contrast estimated. A more reasonable assumption would be to assume a finite number of segregating QTL, with most contrasts representing only random variation, as proposed by Meuwissen et al. [15]. We also analyzed 


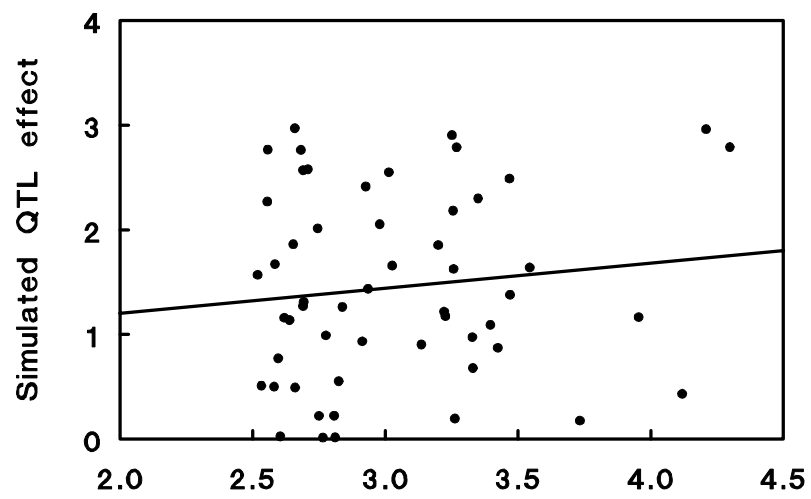

Least squares estimate

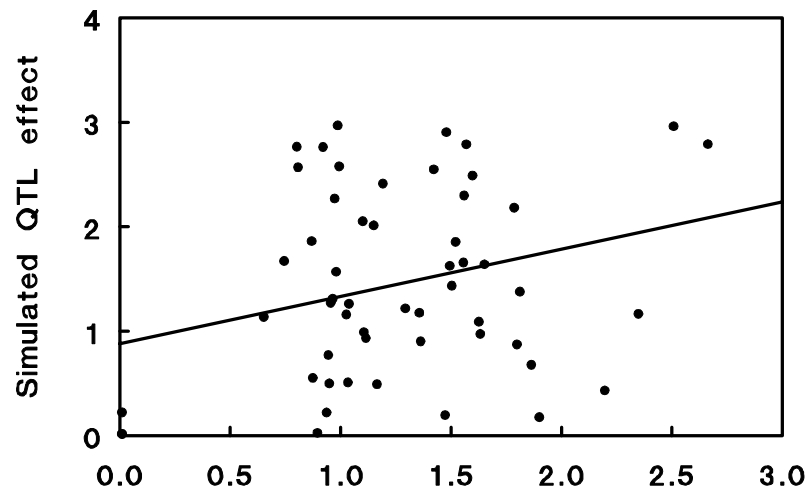

Maximum likelihood estimate
Figure 7. Simulated QTL effects as a function of the least squares estimates.
Figure 8. Simulated QTL effects as a function of the maximum likelihood estimates.

two traits assuming that only a fraction $P$ of the marker contrasts represent segregating QTL, but in both cases the likelihood was maximized with $P$ equal to unity. Another alternative would be to estimate contrasts for marker brackets rather than individual markers. Hayes and Goddard [9] attempted to estimate the number of segregating QTL in the population, but assumed that all "significant" QTL effects represent actual segregating QTL, which is also problematic. Their sample included 50 "significant" contrasts including all families for all traits, while our sample included 490 contrasts for each trait. We estimated the gamma distributions using the contrasts calculated at the marker locations. Even if there is a segregating QTL in linkage to the marker, its effect will be attenuated by recombination, unless the marker and the QTL happen to be tightly linked. However, it can be argued that the practical objective is to 
estimate the distribution of effects that would be detected by a genome scan, not the distribution of true QTL effects.

The equations presented and the simulation studies assumed no segregation distortion, that is the probability that either allele was passed from parents to progeny for all genes is 0.5 . These probabilities could differ from 0.5 due to either meiotic drive or selection. Georges et al. [7] noted that the bulls currently analyzed in their granddaughter design were a selected sample with respect to milk production traits. Analysis of the effect of segregation distortion warrants further study.

Finally, we assumed a common value for $\sigma_{x}$ for each trait, even though the sample sizes varied between traits. Alternatively it should be possible to estimate the parameters of the gamma distribution with each QTL contrast weighted by its specific sample size. Hayes and Goddard [9] also assumed a single value for $\sigma_{x}$, the mean value of the SE as listed in the experiments analyzed to estimate $\mathrm{n}\left(\hat{x}_{i} \mid x\right)$; and assumed that this value was known without error. We estimated this parameter in addition to $\alpha$ and $\beta$, and found that the estimates were generally close to unity, as expected.

Although methods to account for bias in estimating QTL effects have been proposed previously $[1,17,24]$ none are complete solutions. The method of Utz et al. [20] is based on cross validation on independent samples, which is very often not a viable option. Both [1] and [26] dealt chiefly with the problem of bias due to selection. Neither study attempted to correct for the multiple comparison problem, and neither study considered the distribution of QTL effects. Allison et al. [1] noted that his method, which requires significant simulation for each QTL effect may be impractical, if a large number of QTL are estimated. Xu [26] noted that his method is very sensitive to the sample size, and cannot be applied when the sample size is small.

Hayes and Goddard [9] assumed a common gamma distribution of QTL effects for all traits. The current results indicate that this assumption may be problematic. Although the distributions in Figure 1 appear quite different, the confidence intervals for the estimates of $\alpha$ and $\beta$ overlap. As predicted by Weller et al. [24] it should be possible to detect more QTL for traits with higher heritabilities. Since Hayes and Goddard [9] considered only significant effects, their sample was apparently weighted towards the traits with higher heritabilities. Houle et al. [13] discuss various explanations for differences in genetic variances among traits, and conclude that genetic coefficients for life history trait are greater than those for morphological traits. Although they considered numerous traits from seven species, no agricultural animal species were considered, and very little is known about mutation rates for quantitative traits in 
commercial animal species. Differences in genetic variances most likely reflect different selection histories, and environmental variances.

Previous studies $[3,7,9]$ predicted that the bias of QTL effects should decrease as the magnitude of the effect increases. The analyses of the actual data tend to support this conclusion, but not the analysis of the simulated data. Although the mean of the ML estimates was significantly lower than that of the LS estimates, the regression of the ML estimates on the LS estimates was linear and nearly equal to unity. However, no very large QTL effects were generated by the simulation.

Although the proposed estimation of the QTL distribution parameters is rather time-consuming, it need only be done once for each trait for each species. Once the parameters of these distributions have been estimated, ML estimation of the individual QTL effects is straightforward, and the additional computing time is insignificant. A similar strategy is generally applied for commercial genetic evaluations. Variance components are first estimated by REML, and then the estimated values are used for routine genetic evaluations derived by BLUP.

\section{ACKNOWLEDGEMENTS}

This research was supported by a grant from the Israel Milk Marketing Board.

\section{REFERENCES}

[1] Allison D.B., Fernandez J.R., Heo M., Zhu S., Etzel C., Beasley T.M., Amos C.I., Bias in estimates of quantitative trait locus effect in genome scans: demonstration of the phenomenon and a methods-of-moments procedure for reducing bias, Am. J. Hum. Genet. 70 (2002) 575-585.

[2] Beavis W.D., The power and deceit of QTL experiments: lessons from comparative QTL studies, Proceedings of the Forty-Ninth Annual Corn \& Sorghum Industry Research Conference, American Seed Trade Association, Washington, DC, 1994, pp. 250-266.

[3] Beavis W.D., QTL analyses: power, precision, and accuracy, in: Molecular Dissection of Complex Traits, Paterson A.H. (Ed.), CRC Press, New York, 1998, pp. $145-162$.

[4] Broman K.W., Review of statistical methods for QTL mapping in experimental crosses, Lab. Animal 30 (2001) 44-52.

[5] Fernando R.L., Grossman M., Marker assisted selection using best linear unbiased prediction, Genet. Sel. Evol. 21 (1989) 467-477. 
[6] Fernando R.L., Nettleton L.D., Southey B.R., Dekkers J.C.M., Rothschild M.F., Soller M., Controlling the proportion of false positives in multiple dependent tests, Genetics 166 (2004) 611-619.

[7] Georges M., Nielsen D., Mackinnon M., Mishra A., Okimoto R., Pasquino A.T., Sargent L.S., Sorensen A., Steele M.R., Zhao X., Womack J.E., Hoeschele I., Mapping quantitative trait loci controlling milk production in dairy cattle by exploiting progeny testing, Genetics 139 (1995) 907-920.

[8] Goring H.H., Terwilliger J.D., Blangero J., Large upward bias in estimation of locus-specific effects from genomewide scans, Am. J. Hum. Genet. 69 (2001) 1357-1369.

[9] Hayes B., Goddard M.E., The distribution of the effects of genes affecting quantitative traits in livestock, Genet. Sel. Evol. 33 (2001) 209-229.

[10] Henderson C.R., Sire evaluation and genetic trends, in: Proc. Anim. Breed. Genet. Symp. in Honor of Dr. D.L. Lush, ASAS and ADSA, Champaign, IL, 1973, pp. 10-41.

[11] Hoeschele I., Van Raden P.M., Bayesian analysis of linkage between genetic markers and quantitative trait loci. I. Prior knowledge, Theor. Appl. Genet. 85 (1993) 953-960.

[12] Hoeschele I., Van Raden P.M., Bayesian analysis of linkage between genetic markers and quantitative trait loci. II. Combining prior knowledge with experimental evidence, Theor. Appl. Genet. 85 (1993) 946-952.

[13] Houle D., Morikawa B., Lynch M., Comparing mutational variabilities, Genetics 143 (1996) 1467-1483.

[14] Knott S.A., Elsen J.M., Haley C.S., Methods for multiple-marker mapping of quantitative trait loci in half-sib populations, Theor. Appl. Genet. 93 (1996) 71-80.

[15] Meuwissen T.H.E., Hayes B.J., Goddard M.E., Prediction of total genetic value using genome-wide dense marker maps, Genetics 157 (2001) 1819-1829.

[16] Ron M., Blank Y., Band M., Ezra E., Weller J.I., Misidentification rate in the Israeli Dairy cattle population and its implications for genetic improvement, J. Dairy Sci. 79 (1996) 676-681.

[17] Ron M., Kliger D., Feldmesser E., Seroussi E., Ezra E., Weller J.I., Multiple QTL analysis of bovine chromosome 6 in the Israeli Holstein population by a daughter design, Genetics 159 (2001) 727-735.

[18] Ron M., Feldmesser E., Golik M., Tager-Cohen I., Kliger D., Reiss V., Domochovsky R., Alus O., Seroussi E., Ezra E., Weller J.I., A complete genome scan of the Israeli Holstein population for quantitative trait loci by a daughter design, J. Dairy Sci. 87 (2004) 476-490.

[19] Settar P., Weller J.I., Genetic analysis of cow survival in the Israeli dairy cattle population, J. Dairy Sci. 82 (1999) 2170-2177.

[20] Utz H.F., Melchinger A.E., Schon C.C., Bias and sampling error of the estimated proportion of genotypic variance explained by quantitative trait loci determined from experimental data in maize using cross validation and validation with independent samples, Genetics 154 (2000) 1839-1849.

[21] Weller J.I., Comparison of multitrait and single-trait multiple parity evaluations by Monte Carlo simulations, J. Dairy Sci. 69 (1986) 493-500. 
[22] Weller J.I., Quantitative trait loci analysis in animals. CABI Publishing, London, 2001.

[23] Weller J.I., Ezra E., Genetic analysis of the Israeli Holstein dairy cattle population for production and non-production traits with a multitrait animal model, J. Dairy Sci. 87 (2004) 1519-1527.

[24] Weller J.I., Kashi Y., Soller M., Power of "daughter" and "granddaughter" designs for genetic mapping of quantitative traits in dairy cattle using genetic markers, J. Dairy Sci. 73 (1990) 2525-2537.

[25] Weller J.I., Song J.Z., Heyen D.W., Lewin H.A., Ron M., A new approach to the problem of multiple comparisons in the genetic dissection of complex traits, Genetics 150 (1998) 1699-1706.

[26] Xu S., Theoretical basis of the Beavis effect, Genetics 165 (2003) 2259-2268.

To access this journal online: www.edpsciences.org 